\author{
John Leggett ${ }^{1}$ \\ University of Melbourne, \\ Melbourne, Australia \\ e-mail: jake.leggett@unimelb.edu.au \\ Edward Richardson \\ University of Southampton, \\ Southampton, UK \\ e-mail: e.s.richardson@soton.ac.uk \\ Stephan Priebe \\ GE Global Research, \\ Niskayuna, NY \\ e-mail: Stephan.Priebe@ge.com \\ Aamir Shabbir \\ GE Aviation, \\ Cincinnati, $\mathrm{OH}$ \\ e-mail: Aamir.Shabbir@ge.com \\ Vittorio Michelassi \\ General Electric Oil\&Gas, \\ Florence, Italy \\ e-mail: vittorio.michelassi@bhge.com \\ Richard Sandberg \\ University of Melbourne, \\ Melbourne, Australia \\ e-mail: richard.sandberg@unimelb.edu.au
}

\section{Loss Analysis of Unsteady Turbomachinery Flows Based on the Mechanical Work Potential}

Loss analysis is a valuable technique for improving the thermodynamic performance of turbomachines. Analyzing loss in terms of the "mechanical work potential" (Miller, R.J., ASME Turbo Expo 2013, GT2013-95488) provides an instantaneous and local account of the thermal and aerodynamic mechanisms contributing to the loss of thermodynamic performance. This study develops the practical application of mechanical work potential loss analysis, providing the mathematical formulations necessary to perform loss analysis using practical Reynolds-averaged Navier-Stokes (RANS) or large eddy simulations (LES). The analysis approach is demonstrated using RANS and LES of a linear compressor cascade, both with and without incoming wakes. Spatial segmentation is used to attribute loss contributions to specific regions of the flow, and phase-averaging is performed in order to associate the variation of different loss contributions with the periodic passage of wakes through the cascade. For this un-cooled linear cascade, viscous dissipation is the dominant source of loss. The analysis shows that the contribution of the viscous reheat effect depends on the operating pressure of the compressor stage relative to the ambient "dead state" pressureimplying that the optimal blade profile for a low-pressure compressor stage may be different from the optimal profile for a high-pressure compressor stage in the same engine, even if the operating conditions for both stages are dynamically similar. [DOI: 10.1115/1.4048162]

Keywords: computational fluid dynamics (CFD), fluid dynamics and heat transfer phenomena in compressor and turbine components of gas turbine engines, measurement techniques

\section{Introduction}

The drive for energy and propulsion systems that satisfy competing demands for improved efficiency, increased loading, and robust operation has motivated both optimization of conventional turbomachinery components and innovation in turbomachinery systems. Improvement of both turbo-compressors and turbines benefits substantially from careful unsteady aerodynamic design. Many turbomachinery systems of current research interest, including pulsed turbochargers, coupled pressure-gain combustors and turbines, and rotating detonation wave engines are also inherently unsteady and must be analyzed accordingly. In recent years, highfidelity simulation [1,2] and experimental diagnostics [3] have been developed in order to provide detailed information about the behavior and performance of unsteady aerodynamics in turbomachines. For these simulations and measurements to provide actionable insight that benefits the design and development process, it is important to develop correspondingly rigorous techniques for interpreting the unsteady aerodynamic and thermal phenomena present in the high-fidelity data, such as loss analysis.

Loss analysis is concerned with quantifying the conversion of useful energy into forms of energy that cannot be used. The forms of energy that are useful depend on the application. Analysis of external aerodynamic systems typically is concerned with losses of kinetic energy [4]. Analysis of thermal power plant is concerned with the loss of the energy that could be converted into work by reversible processes that exchange heat and work with an environment defined as the "dead state;" this measure of energy is known as "exergy" [5]. In the context of an aerospace propulsion system, it might not be practical to exchange heat with the environment, so

\footnotetext{
${ }^{1}$ Corresponding author.

Contributed by the International Gas Turbine Institute (IGTI) of ASME for publication in the Journal of Turbomachinery. Manuscript received February 5, 2020; final manuscript received May 6, 2020; published online October 16, 2020. Assoc. Editor: Dr. David G. Bogard.
}

the useful energy is the portion of energy that could be converted into work by expanding the working fluid reversibly and adiabatically to the "dead state" pressure: this property has been defined recently by Miller [6] and described as the "mechanical work potential." The mechanical work potential per unit mass is given by

$$
\mathbf{m} \equiv\left(e-e_{s e}\right)+p_{D}\left(v-v_{s e}\right)+\frac{1}{2} u_{i}^{2}
$$

where subscript se denotes the state following isentropic expansion to the dead state pressure $p_{D}$. The change in internal energy $e-e_{s e}$ represents the work done by the fluid during the expansion, $p_{D}(v-$ $v_{s e}$ ) represents the displacement work done on the environment and thereby wasted, and, adopting the Einstein convention for summation over repeated indices, $\frac{1}{2} u_{i}^{2}$ is the kinetic energy of the fluid. The flow mechanical work potential is given by adding pressure work,

$$
\mathbf{m}_{f}=\mathbf{m}+\frac{1}{\rho}\left(p-p_{D}\right)
$$

Composite flow variables, such as the mechanical work potential, are not readily amenable to direct measurement in turbomachinery flows. Instead, turbomachinery losses have been analyzed more commonly in terms of measurements of stagnation pressure changes. For example, the performance of a compressor cascade is traditionally reported in the form of a stagnation pressure loss coefficient

$$
\omega=\frac{p_{01}-p_{02}}{p_{01}-p_{1}}
$$

where $p_{01}$ and $p_{02}$ are the stagnation pressure at inlet and outlet of the cascade, respectively, and $p_{01}-p_{1}$ is the dynamic head at inlet. The pressure loss coefficient is a global measure of loss, but other techniques have been developed in order to estimate how much loss different regions of the flow contribute to the global loss. For example, Denton [7] proposed a method for quantifying 
contributions to global loss from the pressure and suction-surface boundary layers and from the trailing edge wake, based on the boundary layer and blade thicknesses at the trailing edge. Application of Denton's loss analysis in LES of a compressor cascade is presented in Ref. [2].

Pressure-based loss analysis presents difficulties in flows with properties that vary steeply in time or space, since it becomes challenging to characterize inflow and outflow conditions with a single value. Interpretation of stagnation pressure losses is also complicated in practical situations, where losses are not only due to viscous dissipation, but involve heat transfer, mixing, and chemical reaction. Nonetheless, a great deal of design expertise has been learned empirically in terms of pressure loss analysis. Any new loss analysis techniques should therefore have a clear relationship to pressure-loss analysis.

The need for local and instantaneous analysis of losses, including thermal and aerodynamic contributions, has led to entropy-based analysis [8]. Consideration of the entropy transport equation relates these contributions to entropy changes locally and instantaneously, however entropy in itself is not a measure of useful energy in a turbomachine-motivating use of the mechanical work potential.

Miller derived a transport equation for the mechanical work potential of a perfect gas. Loss analysis based on the mechanical work potential transport equation provides an instantaneous and local breakdown of how the physical mechanisms corresponding to the various terms in the transport equation contribute to the overall loss. The rigorous basis of mechanical work potential analysis means that, in principle, it can be applied in a wide variety of flows which are difficult to understand in terms of either total pressure- or entropy-based analyses, for example flows involving significant unsteadiness, heat transfer, mixing or chemical reaction. Formally, loss analysis based on Miller's transport equation requires access to full-resolution measurement or simulation (i.e., DNS) of thermodynamic variables and molecular transport fluxes. In order to apply mechanical work potential-based loss analysis in the process of designing practical turbomachinery, involving highReynolds number turbulence for which DNS is not feasible, it is important to formulate the mechanical work potential analysis in terms of Reynolds-averaged and filtered properties that are computable using the RANS and LES approaches currently available for turbomachinery simulation.

The objectives of this study are to provide the mathematical formulations necessary and practical techniques for applying mechanical work potential-based loss analysis in turbomachinery design simulations. Given the long-standing use of stagnation pressurebased loss analysis, we also seek to relate the mechanical work potential loss analysis to pressure-based metrics. The report proceeds by setting out the configuration and methods used to produce the wall-resolved LES compressor cascade data used for this investigation; presenting a mechanical work potential analysis framework; and then demonstrating how this framework can be applied in the case of an unsteady compressor cascade flow.

\section{Analysis}

Turbulent simulations using Reynolds-averaged Navier-Stokes and large eddy simulations provide Favre-averaged and Favrefiltered solution variables, respectively. In order to use RANS or LES for mechanical work potential-based loss analysis, it is necessary either to evaluate the Favre-averaged or Favre-filtered mechanical work potential from the solution variables, or to solve a Favre-averaged or Favre-filtered transport equation for the mechanical work potential. The necessary mathematical formulations are introduced here. For simplicity, the formulation is presented for the case of a perfect gas.

2.1 Mechanical Work Potential in Turbulent Flows. We denote Reynolds averaging by an overbar $\bar{\psi}$ and Favre (i.e., density- weighted) averages by a tilde $\tilde{\psi} \equiv \overline{\rho \psi} / \bar{\rho}$. Favre fluctuations are denoted $\psi^{\prime \prime}=\psi-\tilde{\psi}$. Note that the overbar and tilde can alternatively be interpreted as filtered and Favre-filtered quantities such that, in the context of LES with a spatio-temporally homogeneous filter, the transport equations below can be interpreted equivalently as LES or Reynolds-averaged equations. For brevity, the equations are introduced using the language of filtering and LES only, rather than ensemble-averaging and RANS. Quantities evaluated using the solution variables available in the LES simulation are described as computable, and denoted by the accent $\check{\psi}$.

Favre-filtering Eq. (1) and Favre decomposition of the velocity gives

$$
\widetilde{\mathbf{m}}=\overline{\left(1-\left(\frac{T_{s e}}{T}\right)\right)}+\frac{p_{D}}{\bar{\rho}}\left[1-\overline{\left(\frac{\rho}{\rho_{s e}}\right)}\right]+\frac{1}{2}{\widetilde{u_{i}}}^{2}+\frac{1}{2} \widetilde{u_{i}^{\prime \prime 2}}
$$

The last term in Eq. (4) is the specific sub-filter turbulent kinetic energy $k=\frac{1}{2} \widetilde{u_{i}^{\prime \prime 2}}$. Given that turbulent kinetic energy is usually dissipated as thermal energy within the flow, it may be argued that the turbulent kinetic energy should be excluded from the mechanical work potential that would be recovered using an ideal turbine and that the turbulent kinetic energy should be counted as a form of internal energy within the turbulent mechanical work potential definition. In LES of an unsteady flow, however, it is difficult to evaluate the turbulent kinetic energy instantaneously, since the majority of turbulent kinetic energy is "resolved" and cannot be distinguished from the kinetic energy of the ensemble-averaged flow also contained in the term $\frac{1}{2} \tilde{u}_{i}^{2}$ without running numerous statistically independent simulations simultaneously. In order to avoid this difficulty, all turbulent kinetic energy is retained as "useful" energy in this analysis. It is also noted that the treatment of turbulent kinetic energy has little effect on the overall analysis since, in highspeed turbomachinery flows, the turbulent kinetic energy is typically on the order of $1 \%$ of the kinetic energy of the ensembleaveraged flow field.

The terms in Eq. (4) involving $e^{\prime \prime}, k, T_{s e} / T$, and $\rho / \rho_{s e}$ are not computable from the LES solution variables. However, in a perfect gas, fluctuations of $T_{s e} / T$ and $\rho / \rho_{s e}$ can be related to pressure fluctuations using isentropic relations. The relative magnitude of turbulent pressure fluctuations $p^{\prime} / \bar{p}$ scales with the turbulent Mach number squared $M_{t}^{2}$, implying that turbulent fluctuations of $T_{s e} / T$ and $\rho /$ $\rho_{\text {se }}$ can be considered as negligible in flows in which $M_{t}^{2} \rightarrow 0$. Assuming that there is an isentropic relationship between turbulent fluctuations of $e$ and $p$, Taylor expansion in terms of $p^{\prime} / \bar{p}$ shows that the errors in the following approximations,

$$
\begin{gathered}
\left(\widetilde{T_{s e}} \frac{p_{D}}{\bar{p}}\right)^{(\gamma-1) / \gamma}+\mathcal{O}\left(\left[\frac{p^{\prime}}{\bar{p}}\right]^{2}\right) \\
e^{\prime \prime}\left(\frac{T_{s e}}{T}\right)=0+O\left(\left[\frac{p^{\prime}}{\bar{p}}\right]\right)
\end{gathered}
$$

and

$$
\overline{\left(\frac{\rho}{\rho_{s e}}\right)}=\left(\frac{p_{D}}{\bar{p}}\right)^{-1 / \gamma}+\mathcal{O}\left(\left[\frac{p^{\prime}}{\bar{p}}\right]^{2}\right)
$$

are of order $\mathcal{O}\left(p^{\prime} / \bar{p}\right)$ and higher. Assuming an adequate model for the sub-filter turbulent kinetic energy is available, the following formula therefore provides an acceptable approximation for evaluating the filtered mechanical work potential in turbomachinery LES in which $M_{t}<<1$ :

$$
\tilde{\mathbf{m}} \approx \tilde{e}\left[1-\left(\frac{p_{D}}{\bar{p}}\right)^{(\gamma-1) / \gamma}\right]+\frac{p_{D}}{\bar{\rho}}\left[1-\left(\frac{p_{D}}{\bar{p}}\right)^{-(1 / \gamma)}\right]+\frac{1}{2} \bar{\rho} \tilde{u}_{i}^{2}+k
$$


2.2 Loss Coefficients. Performance of turbomachinery blade-rows is commonly expressed in terms of loss coefficients defined in terms of the stagnation pressure drop, as in Eq. (3), and in terms of stage efficiencies. Miller [6] introduced a stage efficiency based on mechanical work potential, allowing the loss of efficiency to be attributed to different thermal and aerodynamic processes. Here, loss coefficients are developed in terms of the rate of mechanical work potential destruction within a system $\dot{\mathbf{m}}_{\text {sys }}$, and we develop an approximation to relate mechanical work potential loss coefficient $\omega_{m f}$ to the total pressure loss coefficient in Eq. (3). For a compressor,

$$
\omega_{m_{f}}=\frac{\dot{\mathbf{m}}_{c o m p}}{\left(p_{o 1}-p_{1}\right) / \rho_{o 1}}
$$

and for a turbine,

$$
\omega_{m_{f}}=\frac{\dot{\mathbf{m}}_{\text {turb. }}}{\left(p_{o 2}-p_{2}\right) / \rho_{o 2}}
$$

In a statistically stationary or periodic flow, the rate of mechanical work potential destruction per unit of mass entering the system can be evaluated by considering the integral of the net flux of mechanical work potential into the system surface over a statistically stationary period or by considering a time average of the volume integral of the mechanical work potential source terms within the system volume:

$$
\dot{\mathbf{m}}_{\text {sys }}=\frac{\int_{\text {boundary }} \overline{\rho \vec{u} \mathbf{m}_{f} \cdot \vec{n}} \mathrm{~d} s}{\int_{\text {inlets }} \overline{\rho \vec{u} \cdot \vec{n}} \mathrm{~d} s}=\frac{\int_{\text {vol }} \overline{\rho \dot{\rho \dot{S}_{m}}} \mathrm{~d} v}{\int_{\text {inlets }} \overline{\rho \vec{u} \cdot \vec{n}} \mathrm{~d} s}
$$

where $\vec{n}$ is the unit vector normal to the system surface (pointing inwards) and $\dot{S}_{m}$ is the net source term for mechanical work potential given by the right-hand side of Eq. (15). The periodic variation of the mechanical work potential loss in periodic flows can be analyzed by phase averaging $\rho \dot{S}_{m}$ in the numerator of Eq. (11), but averaging the denominator over a statistically stationary period. The averaged quantities in Eq. (11) are not directly computable from a RANS or LES solution, and modeling of these terms is discussed in Sec. 2.3.

2.2.1 Relationship to Pressure-Based Loss Coefficient. An approximate relationship between the mechanical work potential loss coefficient and the pressure loss coefficient is derived in Appendix A. Neglecting changes in density and considering small finite changes in thermodynamic properties across the stage denoted by $\Delta$, the destruction of mechanical work within the system is given by

$$
\dot{\mathbf{m}}_{s y s} \approx\left(\frac{p_{D}}{p_{01}}\right)^{(\gamma-1) / \gamma} \frac{\Delta p_{0}}{\rho_{1}}
$$

The mechanical work potential and stagnation pressure loss coefficients can then be estimated, respectively, by

$$
\begin{aligned}
& \omega_{\mathbf{m}, \text { est. }} \approx \omega\left(\frac{p_{D}}{p_{01}}\right)^{(\gamma-1) / \gamma} \\
& \omega_{\text {est. }} \approx \omega_{\mathbf{m}}\left(\frac{p_{D}}{p_{01}}\right)^{-(\gamma-1) / \gamma}
\end{aligned}
$$

Experimental characterization of blade designs is often performed with an inlet pressure different to the expected operating pressure of the engine. Equation (13) can then be used to estimate the mechanical work potential loss coefficient at the intended operating pressure.
2.3 Transport Equations. Miller [6] developed a transport equation for the mechanical work potential in a perfect gas:

$$
\begin{aligned}
& \frac{\partial(\rho \mathbf{m})}{\partial t}+\frac{\partial\left(\rho u_{j} \mathbf{m}_{\mathbf{f}}\right)}{\partial x_{j}}=\frac{\partial}{\partial x_{j}}\left(\sigma_{i j} u_{i}\right) \\
& -\frac{\partial}{\partial x_{j}}\left[\left(1-\frac{T_{s e}}{T}\right) q_{j}\right]+\left(\Delta_{\text {therm }}-\Phi_{\text {visc }}\right) \\
& -\left(1-\frac{T_{s e}}{T}\right)\left(\Delta_{\text {therm }}-\Phi_{\text {visc }}\right)
\end{aligned}
$$

where $\vec{q}$ is the heat flux vector, $\vec{\sigma}$ is the viscous stress tensor, and $\Phi_{v i s c} \equiv \sigma_{i j}\left(\partial u_{i} / \partial x_{j}\right)$ is the viscous dissipation rate. Thermal creation, $\Delta_{\text {therm }}$, is given for perfect gases by

$$
\Delta_{\text {therm }}=\frac{\gamma-1}{\gamma} \frac{\nabla p}{p} \cdot \vec{q}
$$

The transport equation for the Favre-averaged or Favre-filtered specific mechanical work potential is derived in Appendix B. The equation for the averaged mechanical work potential can be arranged in a form similar to the instantaneous equation (15):

$$
\begin{aligned}
& \frac{\partial(\bar{\rho} \widetilde{\mathbf{m}})}{\partial t}+\frac{\partial\left(\bar{\rho} \tilde{u}_{j} \tilde{\mathbf{m}}_{f}\right)}{\partial x_{j}}=\frac{\partial \hat{\sigma}_{i j} \tilde{u}_{i}}{\partial x_{j}}-\frac{\partial \hat{\mathcal{T}}_{j}}{\partial x_{j}} \\
& -\frac{\partial}{\partial x_{j}}\left[\left(1-\frac{\check{T}_{s e}}{\tilde{T}}\right) \check{q}_{j}\right]_{(I I I)}+\check{\Delta}_{\text {therm }(I V)}-\check{\Phi}_{v i s c(V)} \\
& -\left(1-\frac{\check{T}_{s e}}{\tilde{T}}\right)\left(\check{\Delta}_{\text {therm }}-\check{\Phi}_{\text {visc }}\right)_{(V I) \&(V I I)} \\
& -\frac{\partial}{\partial x_{j}}\left[\left(1-\frac{\check{T}_{s e}}{\tilde{T}}\right)\left(\bar{q}_{j}-\check{q}_{j}\right)\right]_{(V I I I)}+\frac{\partial}{\partial x_{j}}\left(\overline{\sigma_{i j} u_{i}}-\hat{\sigma}_{i j} \tilde{u}_{i}\right)_{(I X)}+\mathcal{R}_{(X)}
\end{aligned}
$$

in which the turbulent transport term $\hat{\mathcal{T}}_{j}$ is given by

$$
\hat{\mathcal{T}}_{j}=\bar{\rho} \widetilde{e^{\prime \prime} u_{j}^{\prime \prime}}\left(1-\left(\frac{\check{T}_{s e}}{\tilde{T}}\right)\right)+\overline{p u_{j}^{\prime \prime}}+\frac{1}{2} \bar{\rho} \widetilde{u_{j}^{\prime \prime} u_{i}^{\prime \prime} u_{i}^{\prime \prime}}+\bar{\rho} \tilde{u_{i}} \widetilde{u_{j}^{\prime \prime} u_{i}^{\prime \prime}}
$$

and the residual term $\mathcal{R}$ consists of further unclosed terms that cannot be evaluated from the LES or RANS solution variables,

$$
\begin{aligned}
& \mathcal{R}=\frac{\partial}{\partial x_{j}}\left[\overline{\rho u_{j}^{\prime \prime} e^{\prime \prime}\left(\frac{T_{s e}}{T}-\frac{\check{T}_{s e}}{\tilde{T}}\right)}+p_{D} \overline{u_{j}^{\prime \prime}\left(\frac{\rho}{\rho_{s e}}\right)}\right] \\
& +\frac{\partial}{\partial x_{j}}\left[\left(\overline{\frac{T_{s e}}{T} q_{j}}-\frac{\check{T}_{s e}}{\tilde{T}} \bar{q}_{j}\right)\right] \\
& \overline{\frac{T_{s e}}{T}\left(\Delta_{\text {therm }}-\Phi_{\text {visc }}\right)}-\frac{\check{T}_{\text {se }}}{\tilde{T}}\left(\check{\Delta}_{\text {therm }}-\check{\Phi}_{\text {visc }}\right)
\end{aligned}
$$

Several of the terms on the right-hand side of Eq. (17) are analogous to the terms on the right-hand side of Eq. (15) and Miller discusses their roles [6]. The terms in Eq. (17) identified by Roman numerals are described as follows: (I) shear work, (II) turbulent flux, (III) thermal conduction, (IV) thermal creation, (V) viscous dissipation, (VI) thermal recool, (VII) viscous reheat, (VIII) thermal conduction residual, $(I X)$ shear work residual, and $(X)$ the residual $\mathcal{R}$ as defined in Eq. (19).

While terms (II), (VIII), and (IX) are unclosed, the turbulent fluxes $\widetilde{e^{\prime \prime} u_{j}^{\prime \prime}}, \overline{p u_{j}^{\prime \prime}}$, and $\frac{1}{2} \bar{\rho} u_{j}^{\prime \prime} u_{i}^{\prime \prime} u_{i}^{\prime \prime}+\bar{\rho} \tilde{u}_{i} u_{j}^{\prime \prime} u_{i}^{\prime \prime}$, the shear work residual $\overline{\left(\sigma_{i j} u_{i}\right)}-\check{\sigma}_{i j} \tilde{u}_{j}$ and the $\left(\bar{q}_{j}-\check{q}_{j}\right)$ term in the thermal conduction residual appear in the transport equation for total energy employed in RANS or LES, and therefore, closure models for these terms are already available within the simulation. 
The computable value of the ratio $\overline{T_{s e} / T}$ is defined as

$$
\overline{\left(\frac{T_{s e}}{T}\right)} \equiv \frac{\check{T}_{s e}}{\tilde{T}}=\left(\frac{p_{D}}{\bar{p}}\right)^{\frac{\gamma-1}{\gamma}} .
$$

The computable thermal creation term is given by

$$
\check{\Delta}_{\text {therm }}=-\frac{\gamma-1}{\gamma} \frac{\nabla \bar{p}}{\bar{p}} \check{q}_{j}
$$

where the computable heat flux is given by

$$
\check{q}_{j}=-\kappa(\tilde{T}) \frac{\partial \tilde{T}}{\partial x_{j}}
$$

in which $\kappa$ is the temperature-dependent thermal conductivity.

The computable viscous dissipation is given by

$$
\check{\Phi}_{v i s c}=\check{\sigma}_{i j} \frac{\partial \tilde{u}_{i}}{\partial x_{j}}
$$

The computable viscous stress tensor is

$$
\check{\sigma}_{i j}=\mu_{e f f}\left(2 \tilde{S}_{i j}-\frac{2}{3} \delta_{i j} \tilde{S}_{k k}\right)
$$

in which the mean rate of strain tensor is

$$
\tilde{S}_{i j}=\frac{1}{2}\left(\frac{\partial \tilde{u}_{i}}{\partial x_{j}}+\frac{\partial \tilde{u}_{j}}{\partial x_{i}}\right)
$$

In the RANS and LES simulations presented in this study, turbulent transport is modeled using an eddy-viscosity approach, and the dynamic viscosity in Eq. (24) is given by an effective viscosity $\mu_{\text {eff }}=\mu(T)+\mu e d d y$ including temperature-dependent molecular viscosity and the modeled eddy-viscosity.

Due to the rigorous thermodynamic derivation, the mechanical work potential transport equation can be applied in arbitrarily complex flow geometries. In this study, the methodology is demonstrated in the relatively simple context of a linear periodic cascade; however, the methodology in principle is equally valid in fully three-dimensional turbomachinery flows including, for example, end-wall effects and tip leakage.

In most applications, it is sufficient to evaluate the mechanical work potential equation at a small number of time instants for purposes of obtaining (phase-)averages, in which case the computational effort for loss analysis is negligible when compared with the computational cost of the simulation. In the worst case, if the mechanical work potential is transported as a solution variable, the number of transport equations increases by one, and with explicit numerical methods, the computational cost increases approximately in proportion to the number of transport equations-by a factor of $6 / 5$ in the present LES.

2.4 Spatio-Temporal Segmentation. The mechanical work potential and its transport equation provides local and instantaneous information throughout the entire flow domain. In order to associate losses of mechanical work potential with specific design or flow features, the flow domain is segmented into sub-regions. The user is free to specify their own domain segmentation in order to investigate loss mechanisms in specific regions relevant to the flow they are investigating including, for example, regions of tip-leakage and corner separation. The mechanical work potential source terms are then volume-integrated in each sub-region and either time averaged or, in the case with periodic incoming wakes, phase-averaged. In this study, the analysis region in between the inlet and outlet reference planes is divided into four sub-regions, as illustrated in Fig. 1. The four sub-regions are the pressure- and the suction-surface boundary layers, the "wake" region downstream of the blade trailing edges, and the "channel" region in between the boundary layer regions and upstream of the trailing edge. The edge of the boundary layers is defined using the vorticity limit as given by Leggett et al. [9]. In the case with incoming wakes, the vorticity limit is determined using the time-averaged flow field. The mechanical work potential source contributions are non-dimensionalized by dividing by $\left(p_{01}-p_{1}\right) / \rho_{01}$.

\section{Simulations}

3.1 Configuration. Loss analysis is performed based on LES of a linear compressor cascade for a range of incidence angles, with and without incoming wakes. The blade profile is the NACA 65 V103 compressor aerofoil shown in Fig. 2. The nominal angle of incidence is $42 \mathrm{deg}$ at design conditions. The performance of the NACA 65 compressor cascade has been investigated experimentally by Leipold et al. [10] and Hilgenfeld and Pfitzner [11], and computationally by Leggett et al. [2,9]. Comparison between the LES data of Leggett et al. and the experimental data available are presented in Refs. [2,9] showing close agreement in terms of blade surface pressure profiles, pressure loss coefficient and, in both the steady case and the case with periodic incident wakes, boundary layer thicknesses. Therefore, the present loss analysis is based on the well-validated LES data presented by Leggett et al. [2].

The Reynolds number for the simulations is 300,000 based on the chord length $C$; the inflow Mach number is 0.67 ; and the blade pitch is $0.59 C$. The simulation domain extends $0.5 C$ upstream of the blade leading edges ( $0.6 C$ in the case with incoming wakes) and $1 C$ downstream of the trailing edges. A single blade passage is simulated, with periodic boundary conditions in both the spanwise and pitchwise directions, with a spanwise extent of $0.2 C$, making the simulations representative of the flow behavior at mid-span of a linear cascade where the flow is not significantly affected by end-wall effects.

For the cases without incoming wakes, the inflow is fed with synthetic turbulence with $3.5 \%$ turbulence intensity and a length scale of $0.1 C$, with nominal angles of incidence in the range from $37 \mathrm{deg}$ to $49 \mathrm{deg}$. The case with incoming wakes has a nominal angle of incidence equal to $44 \mathrm{deg}$ and no background synthetic turbulence. The incoming wakes are generated by simulating the flow over

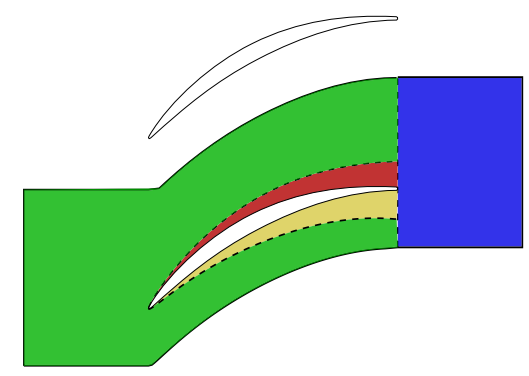

Fig. 1 Schematic outline showing representative volumes for volume breakdown

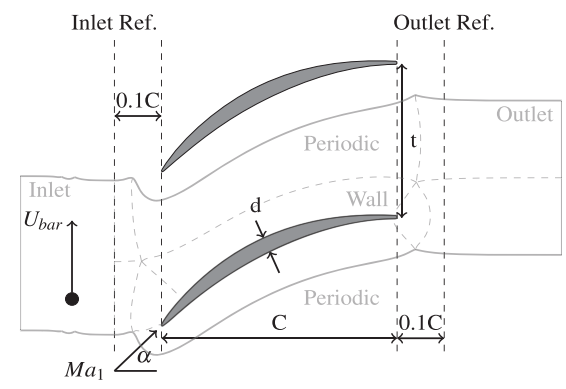

Fig. 2 Schematic of the NACA 65 linear compressor cascade used based on work by Ref. [11]. Computational block boundaries and boundary conditions are shown in light gray. The domain used for loss analysis extends between the inlet and outlet reference planes indicated. 
circular bars passing across the front of the cascade, $0.38 C$ upstream of the blade leading edges. The bar diameter is $0.0595 C$, and the bar pitch is equal to the blade pitch. The bar reduced frequency is set to 1.9 , based on the axial velocity and bar pitch, and its flow coefficient is $u_{b a r} / u_{x}=0.89$. Both the blade and bar boundaries are treated as non-slip and adiabatic.

3.2 Modeling and Numerical Methods. The wall-adaptive local eddy viscosity (WALE) approach [12] is used to close the filtered compressible Navier-Stokes equations. No additional modeling is employed for near-wall turbulence, requiring that the LES mesh resolves near-wall flow structures. The fluid is modeled as a perfect gas with properties of cold air, and the ratio of specific heats equal to 1.4. The synthetic turbulence in the inflow is produced using the digital filter approach of Klein et al. [13].

The LESs are performed using an in-house compressible multiblock structured flow solver, HiPSTAR [14], outlined in greater detail in Ref. [2].

HiPSTAR has previously been used to perform in-depth studies on various subjects such as supersonic wakes [15], unsteady turbulent jets $[16,17]$, and low pressure turbines [14]. Experimental validation for the present axial compressor simulations is reported in Refs. [2,9].

3.3 Computational Setup. The flow around the compressor blade is meshed with an O-grid immersed within an H-grid, as illustrated in Fig. 2. The blade domain is discretized with 603,136 grid points in the cross plane and 128 Fourier modes in the span, giving an effective grid count of $\approx 155 \mathrm{M}$ points. The flow domain around the upstream bars, if present, is meshed similarly, using an O-grid immersed in an $\mathrm{H}$-grid, and connected to the blade domain across a sliding interface, containing a further $\approx 34 \mathrm{M}$ grid points. If upstream bars are not considered, the inflow domain is meshed with two stationary blocks containing $\approx 6 \mathrm{M}$ points. The blade mesh has a wall resolution of $\Delta \mathrm{x}^{+} \approx 15-20, \Delta \mathrm{y}^{+} \approx 1.5$, and $\Delta \mathrm{z}^{+} \approx 12-22$, with the lowest resolution found near the stagnation point of the leading edge. Resolution of the moving bar boundary layers is not of primary importance, since the turbulent wake of the bar develops over several bar widths before interacting with the compressor blades. The resolution of the bar mesh is restricted to the smallest cell size of the blade to ensure no time-step penalty is incurred, resulting in a resolution of $\Delta \mathrm{x}^{+} \approx 5-12, \Delta \mathrm{y}^{+} \approx$ $2-4$, and $\Delta \mathrm{z}^{+} \approx 15-27$ for the bar.

\section{Results}

The loss analysis is presented by first considering the overall loss coefficient and its contributions given by Eq. (17) for all of the "clean" inflow cases without incident wakes and for the moving bar case with moving bars upstream of the cascade. Subsequently, the spatial and spatio-temporal segmentation of the loss contributions is presented for the clean and moving bar cases, respectively. In order to illustrate how the various contributions to the budget depend on the operating pressure of the cascade, relative to the dead state pressure $p_{D}$, each analysis is performed for two values of $p_{01} / p_{D}$. The results for $p_{01} / p_{D}=1$ are indicative of how this compressor cascade would operate in a low-pressure compressor setting and are denoted by the acronym LPC, and the results for $p_{01} / p_{D}=10$ are considered indicative of how this compressor cascade would operate in a higher pressure stage of the compressor and are denoted by HPC. Note that the LPC and HPC results for each inflow case are obtained from a single LES, but post-processed using different values of $p_{D}$.

4.1 Overall Loss Analysis. The mechanical work potential loss coefficients for the clean inlet cases and for the moving bar case are presented in Fig. 3 for the LPC and HPC operating conditions. The mechanical work potential loss coefficient shows a similar variation with incidence at both the LPC and HPC operating conditions, with a minimum close to $44 \mathrm{deg}$ incidence. However, the absolute magnitude of the mechanical work potential loss coefficient is a factor of approximately two greater in the LPC case.

Figure 4 shows that the variation of mechanical work potential loss coefficient in the LPC case has a similar magnitude and variation with angle of incidence as the pressure loss coefficient (note that for the LPC case, $\omega_{e s t}=\omega_{m f}$ since $p_{01}=p_{D}$ ). However, there is a significant divergence between the mechanical work potential loss coefficient and the pressure loss coefficient at more negative values of incidence, indicating that the pressure loss coefficient does not, in general, provide a linear measure of the thermodynamic losses relevant to a propulsion system. The difference between $\omega$ and $\omega_{e s t}$ for the $p_{01}=p_{D}$ LPC case shows the limitations of the simplifying assumptions used to derive Eq. (14). In particular, the divergence between the two loss measures shows the impact on loss prediction due to the spatial correlation between the loss source terms and thermodynamic variables such as static pressure around the blade.

Estimating the pressure loss coefficient based on either the HPC or LPC data using Eq. (14) yields nearly identical values, as shown in Fig. 4. In general, it is not necessary for the two estimates to be equal since the thermal, $p v$, and kinetic energy contributions to the net flux of mechanical work potential have different scaling with $p_{01} / p_{D}$. Put another way, some source terms in the averaged mechanical work potential transport equation (Eq. (17)) have different scaling with $p_{01} / p_{D}$. The very close agreement between the two estimates is therefore remarkable and indicates that in the present simulation cases, although the value of $p_{01} / p_{D}$ may affect the spatial distribution of mechanical work potential source terms, the value $p_{01} / p_{D}$ has little effect on the overall loss following integration of the source terms over the whole flow domain.

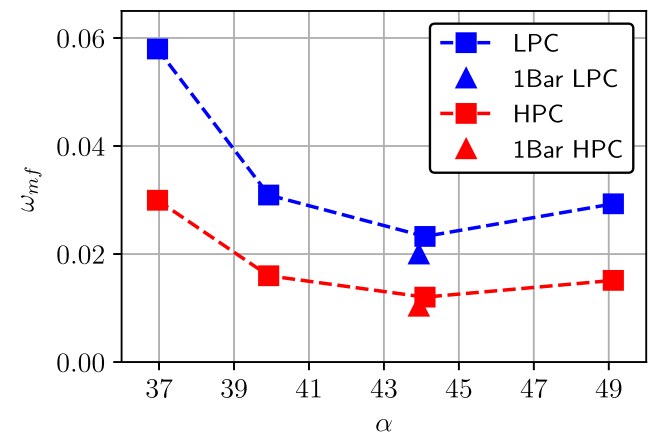

Fig. 3 Mechanical work potential loss coefficient for all cases at both LPC and HPC reference states

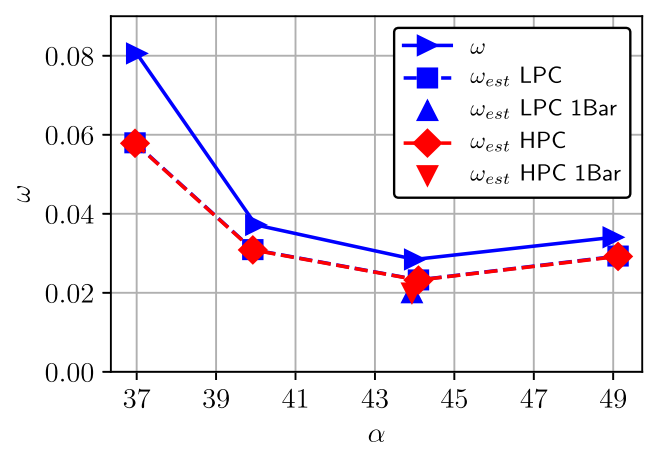

Fig. 4 Estimated pressure loss coefficient calculated using Eq. (14) and mechanical work potential loss coefficient for all cases at both LPC and HPC reference states. Including actual pressure loss coefficient. 


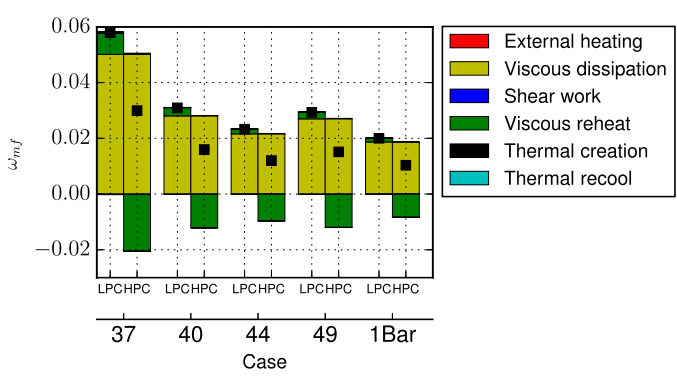

Fig. 5 Mechanical work potential loss breakdown into source terms for all cases at both LPC and HPC reference states. Resultant total loss shown by black square.

The relative importance of the source terms in the averaged mechanical work potential transport equation (Eq. (17)) are assessed by integrating the source terms between the inlet and outlet reference planes. The resulting breakdown of the overall loss is presented in Fig. 5 for each simulation case, at both LPC and HPC conditions. The data are normalized by $\rho_{01} /\left(p_{01}-p_{1}\right)$ in order to show directly how much each term contributes to the mechanical work loss coefficient. The mechanical work loss coefficient given by the sum of source terms in Eq. (17) is indicated by a symbol. Because the simulations address a compressor cascade with no heat transfer at the blade surface, the dominant contribution to the mechanical work potential is associated with viscous dissipation (including both resolved and the modeled sub-filter scale dissipation). Therefore, the only two significant contributions in the mechanical work potential equation are viscous dissipation and viscous reheat.

The present LES simulations are highly resolved with little subfilter variance and loss is dominated by viscous processes. As a result, the magnitudes of the non-viscous terms and other residual terms in Eq. (17) are typically at least two orders of magnitude smaller than the dominant viscous dissipation term and, in the present LES, they make a negligible overall contribution once integrated over the solution domain. RANS simulations for the clean inlet cases without incident wakes were compared with the present LES results in Ref. [9], yielding reasonable overall predictions for pressure loss coefficient, but with some differences in the flow pattern, such as the location of boundary layer transition point on the suction surface and rate of mixing in the wake of the blade. While these RANS simulations are not the focus of the present study, RANS contour plots of viscous dissipation and viscous reheat for the $44 \mathrm{deg}$ HPC case are compared with the corresponding LES results in Fig. 6. The total change in viscous dissipation and viscous reheat between the prescribed reference planes for RANS is found to be -0.02143 and $9.463 \mathrm{e}-3$, respectively, which compares well with the LES results of -0.02159 and 9.551e-3. Despite differences in the flow predictions, it is confirmed that the viscous dissipation and viscous reheat terms (including the modelled dissipation of (sub-filter) turbulent kinetic energy) effectively account for the entire loss of mechanical work potential in both the RANS and LES simulations, with thermal transport terms and other residual terms making negligible overall contributions.

4.2 Spatial Breakdown of Losses. Figure 7 presents the spatial segmentation of the viscous dissipation and viscous reheat loss contributions into the four regions illustrated in Fig. 1. The overall viscous dissipation is dominated by contributions of the boundary layer regions, with minor contributions in the channel and wake regions. The proportion of the loss arising in the suctionsurface boundary layer increases with the angle of incidence, due to thickening of the turbulent boundary layer and growth of the separation bubble shown for the $37 \mathrm{deg}, 44 \mathrm{deg}$, and $49 \mathrm{deg}$ incidence cases in Fig. 8 and discussed in Refs. [2,9]. The spatial distribution of viscous dissipation and viscous reheat for the RANS at $44 \mathrm{deg}$ also compares well with the LES. The suction surface viscous dissipation and viscous reheat are found to be -0.012 and $5.183 \mathrm{e}-3$ compared with the LES -0.012 and $5.075 \mathrm{e}-3$ and the pressure surface viscous dissipation and viscous reheat as $-5.914 \mathrm{e}-3$ and $2.692 \mathrm{e}-3$ compared with the LES $-6.813 \mathrm{e}-3$ and $3.106 \mathrm{e}-3$, respectively.

The viscous dissipation contribution is independent of the dead state pressure $p_{D}$. In contrast, the viscous reheat contribution, as defined in Eq. (17), is affected by the local ratio of static pressure and dead state pressure. The viscous reheat contribution is negative where the local pressure is below the dead state pressure (throughout much of the flow domain for the LPC case) and positive when the static pressure is above the dead state pressure (as in the HPC case).

The relative magnitudes of the viscous reheat contributions from the four different regions of the flow are similar to the relative magnitudes of the viscous dissipation contributions from the corresponding regions, with differences arising due to the variation of static pressure around the blade. Contour plots showing the distribution of static pressure and viscous dissipation are presented in Fig. 8 for the $37 \mathrm{deg}, 44 \mathrm{deg}$, and $49 \mathrm{deg}$ incidence cases. The average static pressure is highest in the pressure-surface boundary layer, followed by the channel, then the wake, and it is lowest in the suctionsurface boundary layer. These differences in static pressure moderate the relative magnitudes of the viscous reheat terms, making the viscous reheat contribution more positive in the pressure-surface boundary layer region, and more negative in the suction-surface boundary layer.

A simple and accurate estimate of the effect of the static pressure distribution on the magnitudes of the viscous reheat contribution for a given region of the flow is given by scaling the viscous dissipation
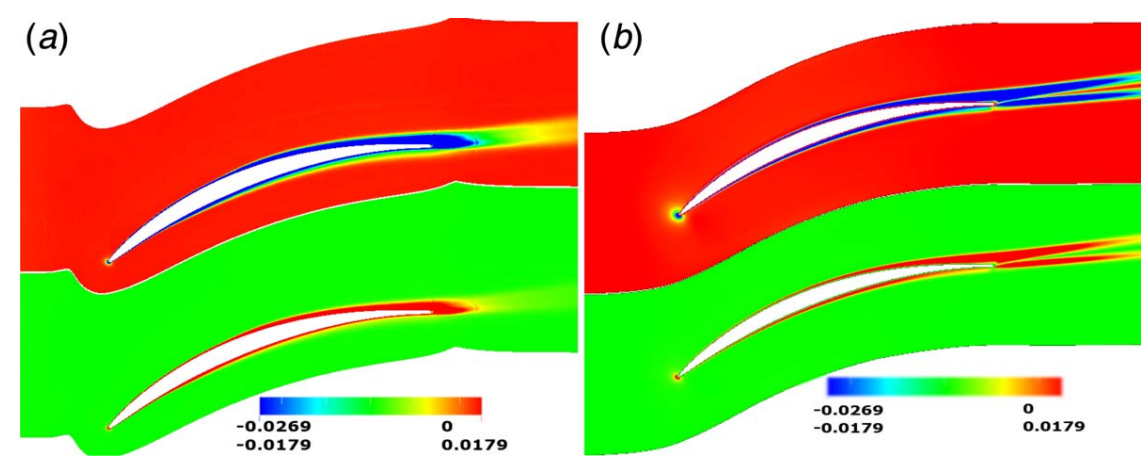

Fig. 6 Color maps showing ensemble-averaged viscous dissipation (top) and viscous reheat (bottom) contributions for (a) LES and (b) RANS simulations. The range of the color maps is $[-0.0269,0]$ for normalized viscous dissipation and $[-0.0179,0.0179]$ for normalized viscous reheat terms. 

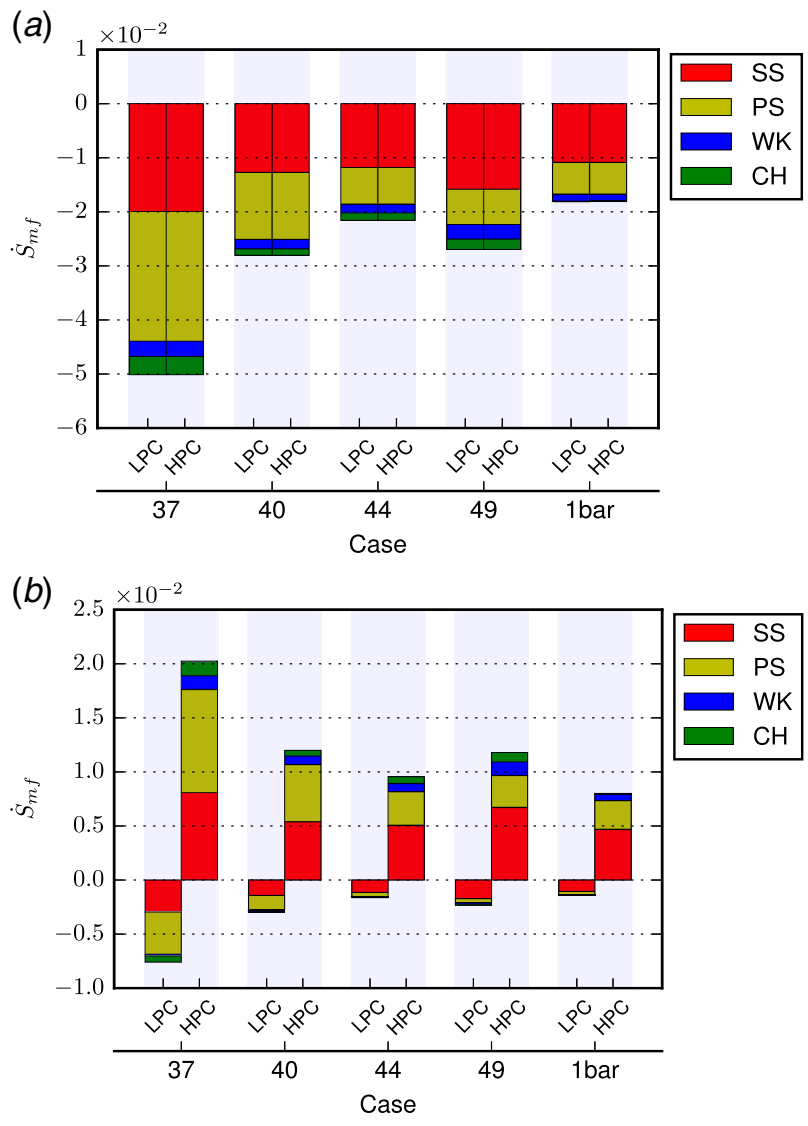

Fig. 7 Volume breakdown of (a) viscous dissipation and (b) viscous reheat for all cases at both LPC and HPC states. Volumes are designated suction surface (SS), pressure surface (PS), wake region (WK), and channel (CH). Normalized with $\left(p_{o 1}-p_{1}\right) / \rho_{o 1}$

contribution by

$$
\frac{\int_{v o l} \overline{\rho \dot{S}_{\text {reheat }}} \mathrm{d} v}{\int_{v o l} \overline{\rho \dot{S}_{\text {diss. }}} \mathrm{d} v} \approx\left(p_{D} / p_{\text {avg }}\right)^{(\gamma-1) / \gamma}-1
$$

where $p_{a v g}$ is the spatially averaged static pressure for that region. Given that, in the $44 \mathrm{deg}$ incidence case, $p_{\text {avg }} / p_{01}=0.786$ for the suction-surface boundary layer and $p_{a v g} / p_{01}=0.877$ for the pressure-surface boundary layer, Fig. 9 shows that the $\left(p_{D} / p_{\text {avg }}\right)^{(\gamma-1) / \gamma}-1$ scaling correctly predicts the ratio of reheat and dissipation contributions for a range of $p_{01} / p_{D}$.

Because the viscous reheat contribution in each region depends on $p_{a v g} / p_{D}$, and because $p_{a v g} / p_{01}$ is constant between dynamically similar operating points (i.e., keeping all non-dimensional numbers other than $p_{01} / p_{D}$ unchanged), the overall performance of a given blade design depends on its operating conditions (i.e., whether it is in a low or high pressure stage, as characterized by $\left.p_{01} / p_{D}\right)$. This implies that the optimal blade design can depend on the target operating condition and that a blade profile optimized based on its performance in an ambient pressure test rig is not necessarily optimal for higher-pressure operation within a gas turbine engine. In particular, this scaling implies that for low-pressure operation, there is a relatively large benefit to localizing viscous dissipation in regions around the blade with relatively high static pressure, potentially at the expense of a small increase of the total viscous dissipation, whereas for higher pressure operation, there is relatively little benefit to localizing the viscous dissipation in higher-pressure regions of the flow, and the primary focus should be on reducing the overall contribution of viscous dissipation.

4.2.1 Denton's Breakdown of Loss. In contrast with the spatial-segmentation of mechanical work potential losses presented here, Denton's 1993 paper [7] proposed a phenomenologically derived approach for quantifying contributions to profile loss from the pressure- and suction-surface boundary layers and from the trailing edge wake of the blade. An advantage of Denton's approach is that the attribution of losses to the different flow regions is based solely on relatively simple measurements of the boundary layer thicknesses and data for the trailing edge blade thickness, whereas the spatial-segmentation of mechanical work potential loss breakdown introduced in this study requires full-field velocity and thermodynamic information that typically is only available from computational fluid dynamics simulation. A limitation of Denton's approach is that loss is attributed to boundary layer properties and not directly to physical processes, for example in order to understand the different contributions of viscous dissipation and viscous reheat.

Denton's analysis has been applied to the clean-inlet LES simulations [2] and the percentage breakdowns by region are compared to the corresponding spatial segmentation of mechanical work potential-based losses in Fig. 10. Denton's method predicts the correct trends, indicating an increase in the relative contribution of losses in the suction-surface boundary layer as the angle of incidence increases. Denton's analysis does not account for losses in the channel region separately, though these losses are relatively small in the present cases. However, the predictions of Denton's method become less accurate moving away from the design incidence, incorrectly suggesting that the pressure-surface loss is almost negligible in the 49 deg incidence case. While Denton's method provides useful information about likely trends in the loss distribution, the magnitude and breakdown of the losses it predicts can give significant errors when compared with the mechanical work potential loss, particularly in the unsteady moving bar case (not shown) where the total loss is almost twice that predicted by the mechanical work potential.
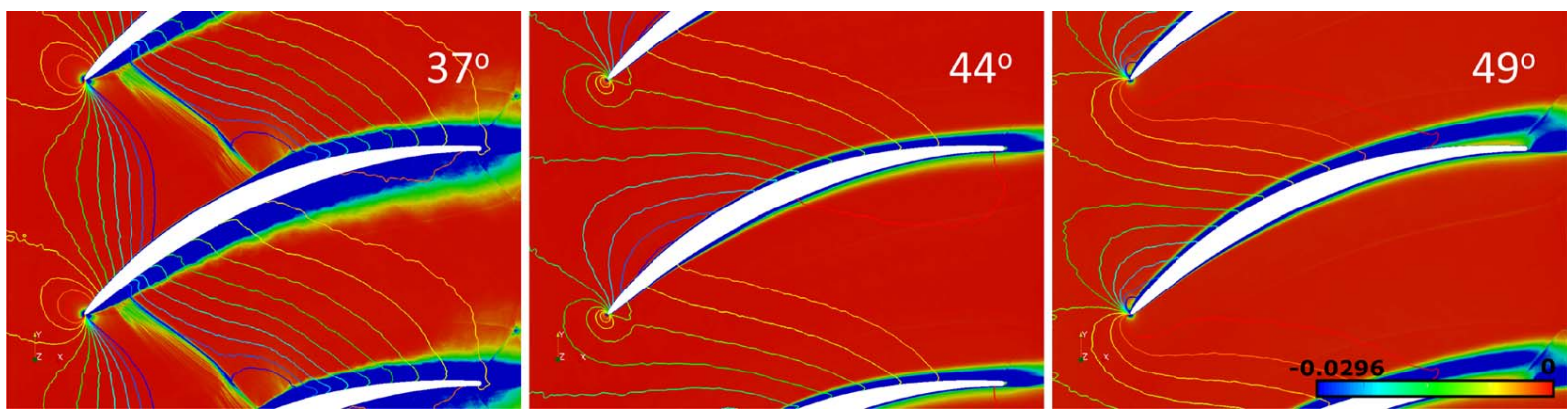

Fig. 8 Ensemble averaged contour plots of viscous dissipation and pressure contours (lines) for the $37 \mathrm{deg}, 44 \mathrm{deg}$, and $49 \mathrm{deg}$ incidence cases. Scale limits from low to high: viscous dissipation $[-0.0269,0], p / p_{01}[0.651,0.884]$. 


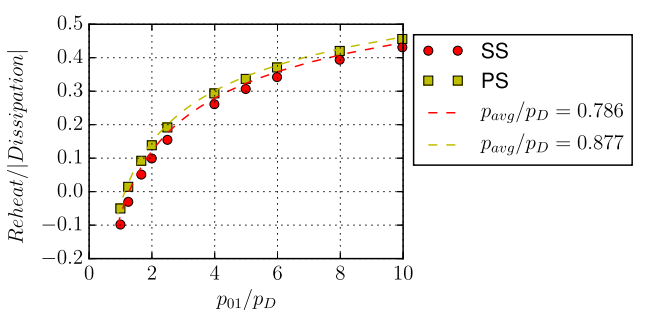

Fig. 9 Ratio of viscous reheat and viscous dissipation versus dead-state pressure for the suction-surface (SS) and pressuresurface (PS) boundary layers of the $\mathbf{4 4} \mathrm{deg}$ case. Dashed-lines show the estimate given by Eq. (26).

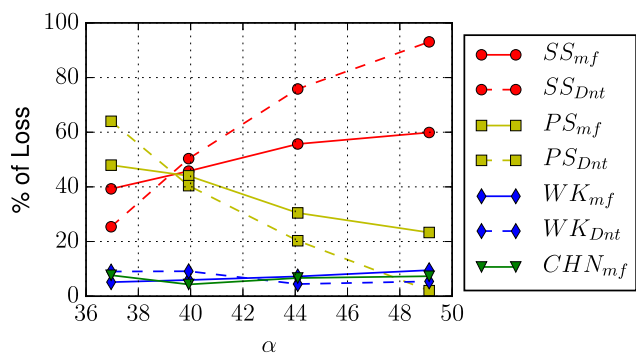

Fig. 10 The fraction of loss contributed by each region of the flow domain, evaluated based on the mechanical work potential analysis (solid) and estimated using Denton's method [7] (dashes). Subscripts, Dnt, are Denton-based breakdown values. The regions defined in Fig. 1 are the suction surface (circles), pressure surface (squares), wake (diamonds), and channel (triangles).

4.3 Spatio-Temporal Breakdown of Losses. The phase-averaged variation of the instantaneous loss coefficient is presented in Fig. 11 for the moving bar case at LPC conditions. The phase is measured from the point in the cycle at which the wake arrives at the leading edge of the blade, as shown in contour plots of ensemble-averaged viscous dissipation and static pressure in Fig. 12. The phase-averaged loss coefficient has an approximately sinusoidal variation with a magnitude approximately equal to $3 \%$ of the time-averaged loss coefficient.

For the operating conditions of the moving bar simulation, the suction surface boundary layer is the dominant source of loss, contributing approximately two-thirds of the overall loss, as shown in Fig. 7. Figure 13 indicates that the phase-averaged loss contribution of the suction-surface boundary layer is in-phase with the overall loss variation. A detailed description of the boundary layer properties and the separate effects of free-stream turbulence and incoming wakes on the present LES cases is given by Leggett et al. [2]. At

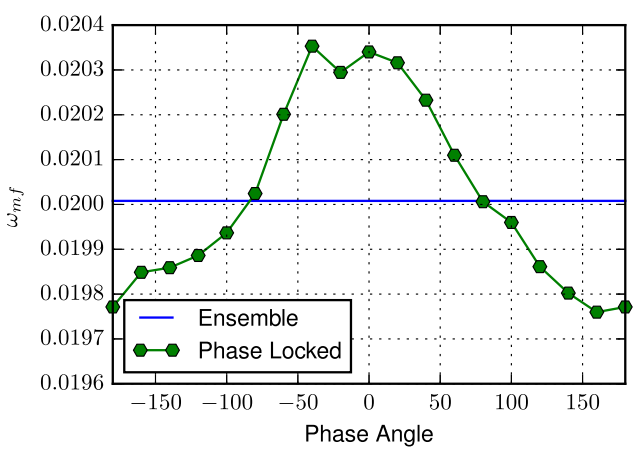

Fig. 11 Phase locked averaged loss over one bar passing period. Including ensemble averaged loss plotted as dashed line.
$44 \mathrm{deg}$ incidence, the suction-surface boundary layer remains laminar until the location of peak suction, a short separation bubble forms above which the boundary layer transitions to turbulence and reattaches. The thick turbulent boundary layer downstream of the separation bubble contributes most of the viscous dissipation. As incoming wakes sweep across the suction-surface boundary layer, the footprint of the wake energizes the laminar boundary layer, promoting earlier transition that periodically eliminates the separation bubble. The portion of boundary layer fluid that does not undergo separation remains more closely attached to the blade as it convects to the trailing edge of the blade, and this portion of fluid contributes less loss than the boundary layer fluid that does not interact with the wakes. The overall effect is that the flow with incident wakes has a lower overall loss coefficient than the 44 deg case without incident wakes (Fig. 5).

The reduction in suction-surface loss caused by the wake interaction promoting earlier transition is realized as the well-attached region of boundary layer it produces passes over the downstream third of the blade chord (i.e., the region that usually contributes most loss). The boundary layer fluid convects at velocity that is slightly less than the wake convection velocity in the free-stream; therefore, the maximum reduction in suction-surface loss is observed around a phase angle of $180 \mathrm{deg}$, approximately when the wake arrives at the trailing edge of the suction-surface.

4.3.1 Wake Recovery. Although the channel region contributes only $6 \%$ of the overall loss, its contribution to the fluctuation of the overall loss has a similar magnitude to the net contribution from the two boundary layer regions. Since wake production by the bars is continuous within the channel region and in this case the bar wakes are largely dissipated by the time they exit from the channel region, though not always the case, the fluctuation of loss within the channel region is due to variation of the bar wake dissipation rate as the bar wakes interact with the pressure field of the blades. It has been confirmed that the periodic thickening of the boundary layers does not cause a significant loss contribution to cross into the channel region.

The interaction of incident wakes with the inter-blade pressure field has been discussed extensively in the context of turbomachinery [18-20] leading to description of the "wake recovery" phenomenon, by which certain measures of stagnation pressure loss are reduced. Wake recovery is due to the overall deceleration of the flow through the channel causing the speed differential between the incident wake and the free-stream fluid to reduce. The wake recovery process is itself inviscid and reversible (and therefore loss-free); however, it can be considered as a loss reduction if one assumes that the wake velocity deficit is eventually mixed out by viscous dissipation. The current definition of mechanical work potential considers that all contributions to kinetic energy (including wake velocity deficits and turbulent eddies) can be recovered as useful work. The inviscid process of wake recovery therefore does not appear directly in the mechanical work potential equation. However the wake recovery process affects the mechanical work potential loss indirectly by affecting the amount of kinetic energy that is subsequently dissipated by viscous processes. In order for mechanical work potential analysis to show wake recovery as a positive source of mechanical work potential, a new "mixed out" definition of mechanical work potential could be considered, which would consider only kinetic energy associated with, for example, the time-averaged flow field as recoverable using Miller's hypothetical ideal turbine, and treating nonrecoverable kinetic energy associated with turbulence and unsteady wakes as unrecoverable. Such a definition would add substantially to the complexity of the mechanical work potential analysis and relies upon a subjective view of what kinetic energy is usable and what is not. However, because the turbulence timescales are short compared to convective timescales in the present simulation case, the effects of wake-recovery rapidly have an effect on the viscous dissipation rate: as the incident wake accelerates toward the location of peak suction on the suction-surface the velocity differential is amplified, leading to maximum loss in the channel from phase angle $-40 \mathrm{deg}$ to $40 \mathrm{deg}$; 

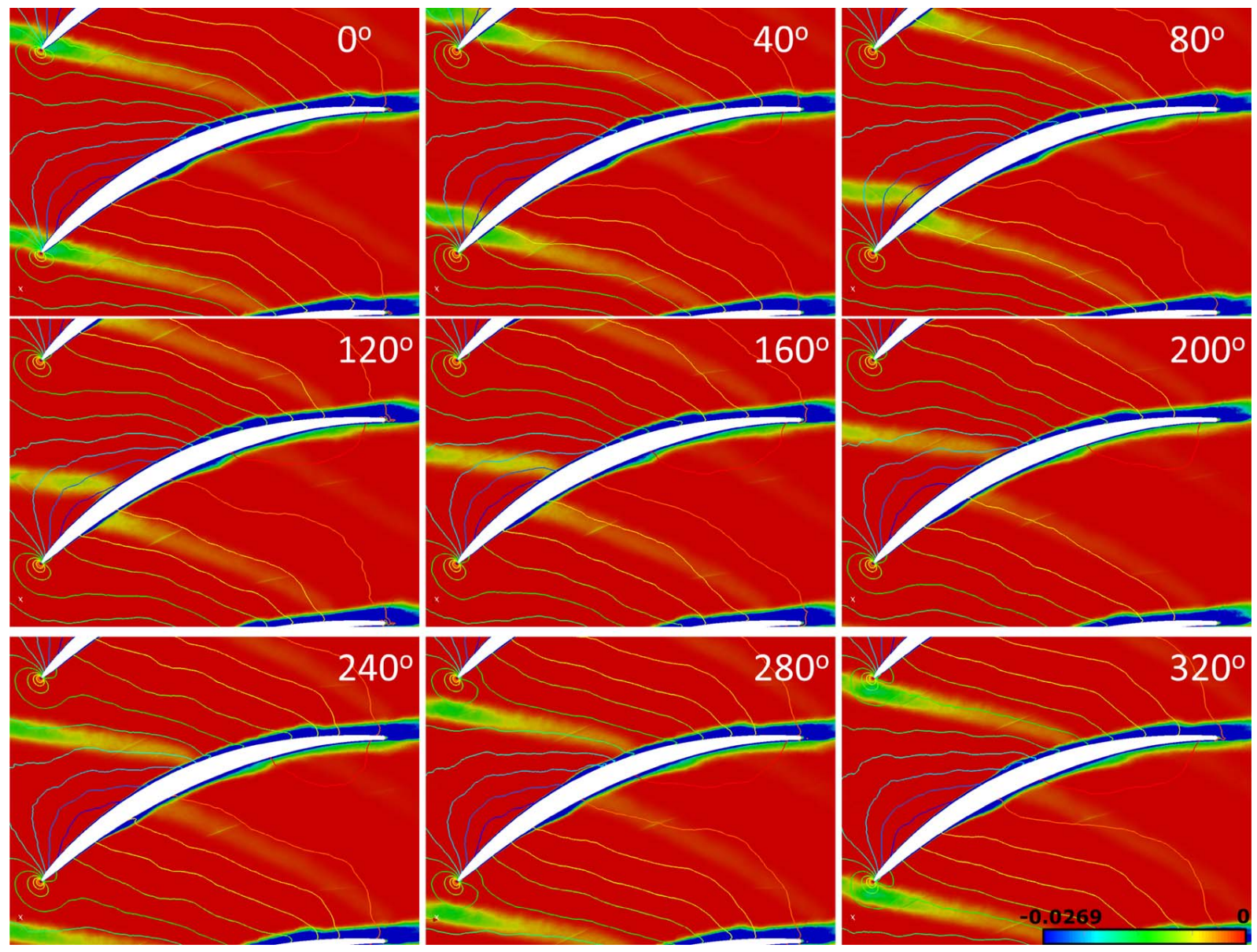

Fig. 12 Contour plots of phase-averaged viscous dissipation and static pressure iso contours from phase angle 0 deg to 320 deg. Scale limits from low to high: viscous dissipation $[-0.0269,0], p / p_{01}[0.651,0.884]$.

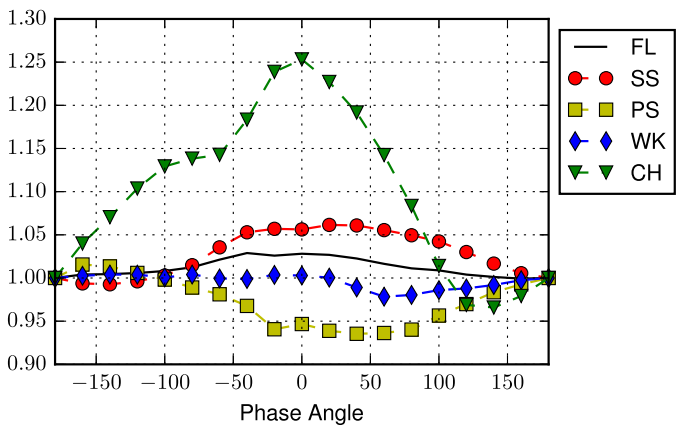

Fig. 13 The periodic variation of the phase-averaged loss in each of the four flow regions shown in Fig. 1. Data are normalized by the values at $\mathbf{- 1 8 0}$ deg. Full field designated as (FL).

and as the velocity differential is attenuated as the incident wake decelerates downstream of the location of peak suction, leading to minimum loss in the channel between phase angles $100 \mathrm{deg}$ to $180 \mathrm{deg}$. The changes in loss are further amplified as the maximum velocity differential occurs at lower pressure resulting in less viscous reheat while the minimum velocity differential has reduced viscous dissipation and hence lower viscous reheat, despite being at a higher pressure.

\section{Conclusions}

This study extends the work of Miller and formulates mechanical work potential analysis for use in practical design simulations based on LES or RANS approaches. It provides an instantaneous filtered/ averaged and local account of the thermal and aerodynamic mechanisms contributing to the loss of propulsive performance. The approach is demonstrated through application to LES and RANS simulations of a linear compressor cascade, leading to the following main conclusions:

- The conventional pressure loss coefficient does not provide a direct measure of the effect of losses on propulsive performance and fail 1 on page 1 to account for how the operating pressure of a particular stage affects its performance within an engine. The derivation of a mechanical work potential loss coefficient and its relationship to the pressure loss coefficient presented in this work highlights this variation of overall loss and the effect of operating pressure is accounted for explicitly.

- Viscous dissipation and the associated viscous reheat effect are the dominant contributions to the overall loss in this un-cooled compressor flow. The contribution of viscous reheat depends on the operating pressure of the compressor stage, implying that, if the flow parameters such as Mach number are constant, the optimal blade geometry for a low-pressure compressor stage may be different from the optimal geometry for a highpressure compressor stage within the same engine due to the change in the ratio of the pressure field to the dead state pressure, resulting in different contributions of viscous reheat from different regions of the flow.

- The mechanical work potential analysis provides an exact spatio-temporal segmentation of losses and their physical origins, and can provide a direct quantification of loss reduction due to flow phenomena such as the early-transition due 
to wake forcing of the boundary layer. In comparison, other methods such as Denton's analysis do not provide direct relation to physical processes and can give significant error under adverse flow conditions.

The mechanical work potential analysis is therefore recommended as a valuable tool within the turbomachine design work-flow.

\section{Acknowledgment}

This work has been performed with support from General Electric and the EPSRC, including grant EP/L002698/1, using resources of the UK National High Performance Computing Facility (ARCHER) EP/L000261/1.

\section{Data Availability Statement}

The datasets generated and supporting the findings of this article are obtainable from the corresponding author upon reasonable request. The authors attest that all data for this study are included in the paper. Data provided by a third party are listed in Acknowledgment. No data, models, or codes were generated or used for this paper.

\section{Nomenclature}

$e=$ internal energy per unit mass

$k=$ turbulent kinetic energy per unit mass

$p=$ static pressure

$t=$ time

$v=$ specific volume

$\mathbf{m}=$ mechanical work potential per unit mass

$T=$ static temperature

$\vec{q}=$ heat flux vector

$\vec{u}=$ velocity vector

$\mathbf{m}_{f}=$ flow mechanical work potential per unit mass

$\vec{x}=$ position vector

$\gamma=$ ratio of isobaric and isochoric specific heat capacities

$\kappa=$ thermal conductivity

$\mu=$ dynamic viscosity

$\rho=$ density

$\vec{\sigma}=$ viscous stress tensor

$\vec{\tau}=$ Reynolds- or sub-grid stress tensor

$\Delta_{\text {therm }}=$ rate of thermal creation

$\Phi_{v i s c}=$ rate of dissipation per unit volume

$\omega=$ loss coefficient

\section{Subscripts}

$$
\begin{aligned}
D & =\text { dead state } \\
0 & =\text { stagnation property } \\
s e & =\text { exit of isentropic turbine expanding to pressure } p_{D} \\
\text { therm } & =\text { thermal } \\
\text { visc } & =\text { viscous }
\end{aligned}
$$

\section{Averaging}

$$
\begin{aligned}
& \bar{\psi}=\text { ensemble average or filtering operation } \\
& \widetilde{\psi}=\text { density-weighted averaging or filtering } \\
& \breve{\psi}=\text { computable quantity }
\end{aligned}
$$

\section{Appendix A}

An approximate relationship between the mechanical work potential loss coefficient and the pressure loss coefficient for a turbomachinery cascade is derived as follows. Assuming that density is constant across the cascade and that changes in fluid properties are small across the cascade, one may write

$$
T \Delta s=\Delta h-\frac{\Delta p}{\rho} \quad \text { and } \quad T_{0} \Delta s=\Delta h_{0}-\frac{\Delta p_{0}}{\rho}
$$

where $\Delta$ represents the difference in a given property between the inlet and outlet of the cascade. The flow mechanical work potential of the fluid is given by the enthalpy change as it expands isentropically through a steady-flow turbine from the stagnation conditions to the dead state pressure:

$$
\mathbf{m}_{f}=h_{0}-h_{s e}
$$

The change in mechanical work potential through the blade row is

$$
\Delta \mathbf{m}_{f}=\Delta h_{0}-\Delta h_{s e}
$$

The change in stagnation enthalpy through a stationary blade row is $\Delta h_{0}=0$, giving $\Delta s=-\Delta p_{0} /\left(\rho T_{0}\right)$. From Eq. (A1), the change $\Delta h_{s e}$ is $T_{s e} \Delta s+\Delta p_{D} / \rho$. Noting that $\Delta p_{D}=0$, substitution into Eq. (A3) gives

$$
\Delta \mathbf{m}_{f} \approx \frac{T_{s e}}{T_{0}} \frac{\Delta p_{0}}{\rho}
$$

Assuming the working fluid is a perfect gas, $T_{s e} / T_{0}=\left(p_{D} / p_{0}\right)^{(\gamma-1) / \gamma}$, giving

$$
\Delta \mathbf{m}_{f} \approx\left(\frac{p_{D}}{p_{01}}\right)^{(\gamma-1) / \gamma} \frac{\Delta p_{0}}{\rho_{1}}
$$

Noting that $\Delta \mathbf{m}_{f}$ in a system with uniform inlet and outlet properties is equal to the rate of mechanical work potential destruction per unit mass entering the system, $\dot{\mathbf{m}}_{\text {sys }}$, the mechanical work potential loss coefficient, $\omega_{m}=\rho_{01} \dot{\mathbf{m}}_{s y s} /\left(p_{01}-p_{1}\right)$, can be related to the pressure loss coefficient, $\omega=\left(p_{01}-p_{02}\right) /\left(p_{01}-p_{1}\right)$ by

$$
\omega_{m} \approx \omega\left(\frac{p_{D}}{p_{01}}\right)^{(\gamma-1) / \gamma}
$$

\section{Appendix B}

The transport equations for the Favre-averaged or Favre-filtered mechanical work potential are obtained respectively by averaging or filtering Eq. (15), giving

$$
\begin{aligned}
& \frac{\partial(\bar{\rho} \tilde{\mathbf{m}})}{\partial t}+\frac{\partial}{\partial x_{j}}\left(\overline{\rho u_{j} \mathbf{m}_{\mathbf{f}}}\right)=\frac{\partial}{\partial x_{j}}\left(\overline{\sigma_{i j} u_{i}}\right) \\
& -\frac{\partial}{\partial x_{j}}\left[\overline{\left(1-\frac{T_{s e}}{T}\right) q_{j}}\right]+\left(\overline{\Delta_{\text {therm }}}-\overline{\Phi_{\text {visc }}}\right) \\
& -\overline{\left(1-\frac{T_{s e}}{T}\right)\left(\Delta_{\text {therm }}-\Phi_{\text {visc }}\right)}
\end{aligned}
$$

The convective flux is decomposed as

$$
\overline{\rho u_{j} \mathbf{m}_{f}}=\bar{\rho} \tilde{u}_{j} \tilde{\mathbf{m}}_{f}+\mathcal{T}_{j}
$$

Splitting $\mathcal{T}_{j}$ the turbulent flux of flow mechanical work potential, into internal energy, $p v$, and kinetic energy contributions, as in Eqs. (1) and (2), gives

$$
\begin{aligned}
\mathcal{T}_{j}=\bar{\rho} \widetilde{u_{j}^{\prime \prime} \mathbf{m}_{f}^{\prime \prime}=} & \bar{\rho} u_{j}^{\prime \prime} e^{\prime \prime}\left(\widetilde{\left.1-\left(\frac{T_{s e}}{T}\right)\right)}-\bar{\rho} \tilde{e} u_{j}^{\prime \prime}\left(\frac{T_{s e}}{T}\right)\right. \\
& +\overline{p u_{j}^{\prime \prime}}-p_{D} \overline{u_{j}^{\prime \prime}\left(\frac{\rho}{\rho_{s e}}\right)}+\frac{1}{2} \bar{\rho} \widetilde{u_{j}^{\prime \prime} u_{i}^{\prime \prime} u_{i}^{\prime \prime}}+\bar{\rho} \tilde{u_{i}} \widetilde{u_{j}^{\prime \prime} u_{i}^{\prime \prime}} .
\end{aligned}
$$

Several terms in Eqs. (B1) and (B3) are not computable from the RANS or LES solution variables. In order to develop a form of Eq. (B1) that can be modeled and computed from RANS or LES solutions, the various terms are split into a computable part and a residual part. The computable quantities $\overline{T_{s e} / T}, \check{\Delta}_{\text {therm }}, \check{q}_{j}$, and $\check{\Phi}_{\text {visc }}$ and $\check{\sigma}_{i j}$ are defined in Eqs. (20)-(24). The turbulent flux expression given in Eq. (B3) can also be decomposed further, separating out the terms that define $\hat{\mathcal{T}}_{j}$ in Eq. (18) which appear in the averaged 
or filtered total energy transport equation and are therefore the subject of models already employed in the RANS or LES computation. Unclosed and un-modelled terms in Eq. (B1) are relegated to a residual term $\mathcal{R}$ defined in Eq. (19), yielding the final transport equation for the averaged mechanical work potential, given as Eq. (17).

\section{References}

[1] Michelassi, V, Chen, L. W., Pichler, R., and Sandberg, R. D., 2015, "Compressible Direct Numerical Simulation of Low-Pressure Turbines-Part II: Effect of Inflow Disturbances," ASME J. Turbomach, 137(7), p. 071005.

[2] Leggett, J, Priebe, S, Shabbir, A, Michelassi, V, Sandberg, R. D., and Richardson, E, 2018, "Loss Prediction in an Axial Compressor Cascade at Off-Design Incidences With Free Stream Disturbances Using Large Eddy Simulation," ASME J. Turbomach, 140(7), p. 071005

[3] Sinkwitz, M, Winhart, B, Engelmann, D, Mare, F di, and Mailach., R, 2018, "On the Periodically Unsteady Interaction of Wakes, Secondary Flow Development and Boundary Layer Flow in an Annular LPT Cascade: Part 1-Experimental Investigation," ASME Turbo Expo 2018: Turbomachinery Technical Conference and Exposition, Oslo, Norway.

[4] Drela, M, 2009, "Power Balance in Aerodynamic Flows," AIAA J., 47(7), pp. 1761-1771.

[5] Dunbar, W. R., Lior, N, and Gaggioli, R. A., 1992, "The Component Equations of Energy and Exergy," ASME J. Energy Res. Technol., 114(1), pp. 75-83.

[6] Miller., R. J., 2013, "Mechanical Work Potential," ASME Turbo Expo 2013, San Antonio, TX, pp. 1-13.

[7] Denton, J. D., 1993, "The 1993 IGTI Scholar Lecture: Loss Mechanisms in Turbomachines," ASME J. Turbomach, 115(4), pp. 621-656.

[8] S Wheeler, A. P., Sandberg, R. D., Sandham, N. D., Pichler, R, Michelassi, V, and Laskowski, G, 2016, "Direct Numerical Simulations of a High-Pressure Turbine Vane," ASME J. Turbomach, 138(7), p. 071003.
[9] Leggett, J, Sandberg, R. D., Michelassi, V, Priebe, S, and Shabbir., A, 2016, "Detailed Investigation of Rans and Les Predictions of Loss Generation," ASME Turbo Expo: Power for Land, Sea, and Air, Seoul, South Korea, Vol. 2A, p. 49699.

[10] Leipold, R, Boese, M, and Fottner, L, 2000, "The Influenceof Technical Surface Roughness Caused by Precision Forging on the Flow Around a Highly Loaded Compressor Cascade," ASME J. Turbomach, 122(3), pp. 416-424.

[11] Hilgenfeld, L, and Pfitzner, M, 2004, "Unsteady Boundary Layer Development Due to Wake Passing Effects on a Highly Loaded Linear Compressor Cascade," ASME J. Turbomach, 126(4), pp. 493-500.

[12] Nicoud, F, and Ducros, F, 1999, "Subgrid-Scale Stress Modelling Based on the Square of the Velocity Gradient Tensor," Flow Turbulence Combust, 62(3), pp. 183-200.

[13] Klein, M, Sadiki, A, and Janicka, J, 2003, “A Digital Fil-Ter Based Generation of Inflow Data for Spatially Developing Direct Numerical or Large Eddy Simulations," J. Comput. Phys, 186(2), pp. 652-665.

[14] Sandberg, R. D., Michelassi, V, Pichler, R, Chen, L, and Johnstone, R, 2015, "Compressible Direct Numerical Simulation of Low-Pressure Turbines-Part I: Methodology," ASME J. Turbomach, 137(5), p. 051011.

[15] Sandberg, R. D., 2012, "Numerical Investigation of Turbulent Supersonic Axisymmetric Wakes," J. Fluid Mech, 702(1), pp. 488-520.

[16] Shin, D. H., Aspden, A. J., and Richardson, E. S., 2017, "Self-Similar Properties of Decelerating Turbulent Jets," J. Fluid Mech., 833(1), p. R1.

[17] Shin, D. H., Sandberg, R. D., and Richardson, E. S., 2017, "Self-Similarity of Fluid Residence Time Statistics in a Turbulent Round Jet," J. Fluid Mech, $\mathbf{8 2 3}(1)$, pp. 1-25.

[18] Smith, L. H., 1966, "Wake Dispersion in Turbomachines," J. Basic Engin., 88(3), pp. 688-690.

[19] Praisner, T. J., Clark, J. P., Nash, T. C., Rice, M. J., and Grover, E. A., 2006, "Performance Impacts Due to Wakemixing in Axial-flow Turbomachinery," ASME Turbo Expo 2006: Power for Land, Sea, and Air, Barcalona, Spain, American Society of Mechanical Engineers, pp. 1821-1830.

[20] Hodson, H. P., Hynes, T. P., Greitzer, E. M., and Tan, C. S., 2009, "A Physical Interpretation of Stagnation Pressure and Enthalpy Changes in Un-Steady Flow," ASME J. Turbomach, 134(6), p. 060902. 